\title{
Comment on "Investigation of Possible Herb-Drug Interactions for the Treatment of Cardiovascular Diseases"
}

\author{
Bradley J. McEwen, PhD, MHSc${ }^{1}$ \\ ${ }^{1}$ School of Health and Human Sciences, Southern Cross University, \\ Military Road, Lismore New South Wales, Australia \\ Semin Thromb Hemost 2019;45:552-553.
}

Cardiovascular disease (CVD) is the leading cause of morbidity and mortality worldwide. ${ }^{1}$ CVD is a multifactorial disease that affects a large portion of the population with risk factors including increased platelet aggregation and activation, ${ }^{2}$ increased coagulation, decreased fibrinolytic capacity, ${ }^{3}$ type-2 diabetes, dyslipidemia, being overweight or obese, physical inactivity, poor nutrition, and a family history of CVD. ${ }^{1}$ Cardiovascular disease has been linked to numerous health conditions, such as chronic inflammation ${ }^{1,4}$ increased oxidative stress ${ }^{4,5}$ nonalcoholic fatty liver disease, obstructive sleep apnoea, ${ }^{1}$ and polycystic ovary syndrome. ${ }^{6}$

Diet (e.g., the Mediterranean and vegetarian diet), specific foods (e.g., berries, chocolate, garlic, tomato, and turmeric), ${ }^{7}$ nutrients (such as omega-3), ${ }^{7-9}$ and herbal medicines (e.g., feverfew, willow bark) ${ }^{10}$ alter platelet function. Herbal medicines can also alter coagulation. ${ }^{10}$ Herbal medicines have a long history of worldwide use. They have numerous actions, including antiplatelet, hemostatic, anti-inflammatory, antioxidant, cholesterol lowering, antispasmodic, expectorant, and immunomodulatory properties. ${ }^{10}$

Herbal medicines in Australia are defined as a therapeutic good and are regulated as medicines under the Therapeutic Goods Act 1989. All therapeutic goods must conform to applicable standards recognized under the Act. Australia is one of the few countries in the world to manufacture complementary medicines to pharmaceutical standards and good manufacturing practice (GMP) standards. ${ }^{11}$ Herbal and nutritional medicines are commonly used by patients alongside standard medical therapy. Herbal medicines have the potential to interact with many medications, particularly antiplatelet and anticoagulant agents, such as aspirin, clopidogrel, and warfarin, ${ }^{10}$ and may be prescribed together with conventional therapies.

In their recent letter, Zuo et al discussed the potential mechanism of action and possible interaction of the combination of danshen (Salvia miltiorrhiza) and chuanxiong rhizome (Ligusticum chuanxiong; DS-CX) with conventional drugs. ${ }^{12}$ These two herbal medicines have been utilized for invigorating
Address for correspondence Bradley J. McEwen, PhD, MHSc, School of Health and Human Sciences, Southern Cross University, PO Box 157, Lismore New South Wales 2480, Australia (e-mail: nutrition1@bigpond.com).

blood circulation and eliminating stasis. ${ }^{13,14}$ Based on the target-pathway associations, a "target-(pathway)-target" (TPT) network, the authors investigated the targets of DS - CX and conventional drugs. The particular pathways of this study included platelet activation, vascular smooth muscle contraction, and complement and coagulation cascades. The authors found that the combination of these two herbal medicines interacted with several targets of conventional cardiovascular drugs. They found that the herb-drug interaction between conventional cardiovascular drugs and DS-CX may be more serious in the vascular smooth muscle contraction pathway and the complement and coagulation cascades pathway.

However, for the management of CVDs and other chronic diseases, it is suggested that herbal medicines are best prescribed by health professionals qualified in herbal medicine or by physicians with training in herbal medicine for patients taking medications, particularly antiplatelet or anticoagulation drugs, following a consultation based on the results of in-depth case talking and clinical history. Additionally, patients should be monitored for treatment effect and potential interactions with medications.

In conclusion, herbal medicines play a beneficial role in the management of chronic diseases, especially CVD. Further research in humans is suggested to investigate the effects of herbal medicines on platelet aggregation and function and coagulation. Additionally, research is suggested to investigate their effects on the various other risk factors of CVD, such as inflammation, oxidative stress, atherosclerosis, hyperglycemia, hypercholesterolemia, and hypertriglyceridemia, and for potential interactions.

Conflicts of Interest

There is no conflict of interest to declare.

\section{References}

1 Benjamin EJ, Virani SS, Callaway CW, et al; American Heart Association Council on Epidemiology and Prevention Statistics published online June 13, 2019
Issue Theme Editorial Compilation VII; Guest Editors: Emmanuel J. Favaloro, PhD, FFSc (RCPA), and Giuseppe Lippi, MD.
Copyright $\odot 2019$ by Thieme Medical Publishers, Inc., 333 Seventh Avenue, New York, NY 10001, USA. Tel: +1(212) 584-4662.
DOI https://doi.org/ 10.1055/s-0039-1692212. ISSN 0094-6176. 
Committee and Stroke Statistics Subcommittee. Heart disease and stroke statistics-2018 update: a report from the American Heart Association. Circulation 2018;137(12):e67-e492

2 Ruggeri ZM. Platelets in atherothrombosis. Nat Med 2002;8(11): 1227-1234

3 Koenig W. Haemostatic risk factors for cardiovascular diseases. Eur Heart J 1998;19(Suppl. C):C39-C43

4 Whalen KA, McCullough ML, Flanders WD, Hartman TJ, Judd $\mathrm{S}$, Bostick RM. Paleolithic and mediterranean diet pattern scores are inversely associated with biomarkers of inflammation and oxidative balance in adults. J Nutr 2016;146(06): $1217-1226$

5 Govindarajan G, Whaley-Connell A, Mugo M, Stump C, Sowers JR. The cardiometabolic syndrome as a cardiovascular risk factor. Am J Med Sci 2005;330(06):311-318

6 McEwen BJ. Can omega-3 polyunsaturated fatty acids improve metabolic profile in polycystic ovary syndrome (PCOS)? Adv Integr Med 2017;4(02):82-83

7 McEwen BJ. The influence of diet and nutrients on platelet function. Semin Thromb Hemost 2014;40(02):214-226

8 McEwen BJ, Morel-Kopp MC, Chen W, Tofler GH, Ward CM. Effects of omega-3 polyunsaturated fatty acids on platelet function in healthy subjects and subjects with cardiovascular disease. Semin Thromb Hemost 2013;39(01):25-32

9 McEwen B, Morel-Kopp MC, Tofler G, Ward C. Effect of omega-3 fish oil on cardiovascular risk in diabetes. Diabetes Educ 2010;36 (04):565-584

10 McEwen BJ. The influence of herbal medicine on platelet function and coagulation: a narrative review. Semin Thromb Hemost 2015; 41(03):300-314

11 Australian Government Department of Health Therapeutic Goods Administration. An overview of the regulation of complementary medicines in Australia. Available at: https://www.tga.gov.au/ overview-regulation-complementary-medicines-australia. Accessed May 20, 2019

12 Zuo H, Yang F, Hu Y. Investigation of possible herb-drug interactions for the treatment of cardiovascular diseases. Semin Thromb Hemost 2019;45(05):548-551

13 Chen Z, Zhang C, Gao F, et al. A systematic review on the rhizome of Ligusticum chuanxiong hort. (chuanxiong). Food Chem Toxicol 2018;119:309-325

14 Chen X, Guo J, Bao J, Lu J, Wang Y. The anticancer properties of Salvia miltiorrhiza Bunge (danshen): a systematic review. Med Res Rev 2014;34(04):768-794 